PROCEEDINGS OF THE

AMERICAN MATHEMATICAL SOCIETY

Volume 136, Number 6, June 2008, Pages 1887-1891

S 0002-9939(08)09367-2

Article electronically published on February 11, 2008

\title{
SPLITTING CRITERION FOR REFLEXIVE SHEAVES
}

\author{
TAKURO ABE AND MASAHIKO YOSHINAGA \\ (Communicated by Ted Chinburg)
}

AbStract. We give a criterion for a reflexive sheaf to split into a direct sum of line bundles.

\section{MAIN THEOREM}

Vector bundles over the projective space $\mathbf{P}_{\mathbb{K}}^{n}$ are one of the main subjects in both (algebraic) geometry and commutative algebra. The most fundamental result in this area is the theorem due to Grothendieck which asserts that any holomorphic vector bundle over $\mathbf{P}_{\mathbb{K}}^{1}$ splits into a direct sum of line bundles. When $n \geq 2$, vector bundles over $\mathbf{P}_{\mathbb{K}}^{n}$ do not necessarily split. Indeed, the tangent bundle is indecomposable. In these cases, some sufficient conditions for vector bundles to split have been established. The following is one of such criterions, which we call "Restriction criterion".

Theorem 0.1 (Horrocks). Let $\mathbb{K}$ be an algebraically closed field, $n$ be an integer greater than or equal to 3 , and let $E$ be a locally free sheaf on $\mathbf{P}_{\mathbb{K}}^{n}$ of $\operatorname{rank} r(\geq 1)$. Then $E$ splits into a direct sum of line bundles if and only if there exists a hyperplane $H \subset \mathbf{P}_{\mathbb{K}}^{n}$ such that $\left.E\right|_{H}$ splits into a direct sum of line bundles.

In other words, the splitting of a vector bundle can be characterized by using a hyperplane section. However, vector bundles, or equivalently locally free sheaves, form a small class among all coherent sheaves. There are some important wider classes of coherent sheaves, e.g., reflexive sheaves or torsion free sheaves. The purpose of this article is to generalize the "Restriction criterion" to one for reflexive sheaves, and we also show that it fails in the class of torsion free sheaves. Our main theorem is as follows:

Theorem 0.2. Let $\mathbb{K}$ be an algebraically closed field, $n$ be an integer greater than or equal to 3 , and let $E$ be a reflexive sheaf on $\mathbf{P}_{\mathbb{K}}^{n}$ of rank $r(\geq 1)$. Then $E$ splits into a direct sum of line bundles if and only if there exists a hyperplane $H \subset \mathbf{P}_{\mathbb{K}}^{n}$ such that $\left.E\right|_{H}$ splits into a direct sum of line bundles.

We give two proofs for Theorem 0.2 The first proof is basically parallel to that of Theorem 0.1 in which we also establish a general principle that the structure of a reflexive sheaf can be recovered from its hyperplane section (Theorem 2.2).

Received by the editors May 22, 2006.

2000 Mathematics Subject Classification. Primary 14J60.

Key words and phrases. Splitting criterion, reflexive sheaves, hyperplane arrangement.

(C)2008 American Mathematical Society

Reverts to public domain 28 years from publication 
The second proof is based on a cohomological characterization for a coherent sheaf to be locally free. By using it, the proof is reduced to Theorem 0.1 .

Theorem 0.2 was first proved for special kinds of reflexive sheaves in [8], namely for sheaves of logarithmic vector fields tangent to hyperplane arrangements. Theorem 0.2 is a generalization to any reflexive sheaves. The splitting of these sheaves has some applications to combinatorial problems of hyperplane arrangements. See [6] or [7] for details.

The organization of this article is as follows. In \$1 we recall some basic results on reflexive sheaves from [3. In $\$ 2$ we give the first proof of the main theorem. In \$3. we give the second proof by using cohomological characterizations for a coherent sheaf to be locally free.

\section{Preliminaries}

In this section, we fix the notation and prepare some results for the proof of Theorem 0.2. We use the terms "vector bundle" and "locally free sheaf" interchangeably. The term "variety" means an integral scheme of finite type over a field. Let $X$ be a smooth variety of dimension $n$ over a field $\mathbb{K}$, where $n \geq 1$ and $\mathbb{K}$ is an algebraically closed field. For a coherent sheaf $E$ on $X$, $\operatorname{Sing}(E)$ denotes the non-free locus of $E$, i.e., $\operatorname{Sing}(E):=\left\{x \in X \mid E_{x}\right.$ is not a free $\mathcal{O}_{x, X}$-module $\}$. The dual of a coherent sheaf $E$ (on $X$ ) is denoted by $E^{*}$.

In this article, we employ homological algebra to consider properties of a coherent sheaf on a smooth variety $X$. Let us review some definitions and results. For a coherent sheaf $E$ on $X$ over $\mathbb{K}$ and for a point $x \in X$, define the depth of the $\mathcal{O}_{x, X}$-module $E_{x}$ (denoted by $\operatorname{depth}_{\mathcal{O}_{X}}\left(E_{x}\right)$ ) as the length of a maximal $E_{x}$-regular sequence in $\mathcal{M}_{x}$, where $\mathcal{M}_{x}$ is the unique maximal ideal of a local ring $\mathcal{O}_{x, X}$. Moreover, we define the projective dimension of an $\mathcal{O}_{x, X}$-module $E_{x}$ (denoted by $\left.\operatorname{pd}_{\mathcal{O}_{x, X}}\left(E_{x}\right)\right)$ as the length of a minimal free resolution of $E_{x}$ as an $\mathcal{O}_{x, X}$-module. It is known that every module which is finitely generated over a regular local ring has finite projective dimension. These two quantities are related by the famous Auslander-Buchsbaum formula as follows:

$$
\operatorname{depth}_{\mathcal{O}_{x, X}}\left(E_{x}\right)+\operatorname{pd}_{\mathcal{O}_{x, X}}\left(E_{x}\right)=\operatorname{dim} \mathcal{O}_{x, X} .
$$

Hence it follows easily that a coherent sheaf $E$ on $X$ is locally free if and only if $\operatorname{depth}_{\mathcal{O}_{x, X}}\left(E_{x}\right)=\operatorname{dim} \mathcal{O}_{x, X}$ for all $x \in X$. For details and proofs, see [4]. The projective dimension can also be characterized as follows (for example, see [5], Chapter II):

Lemma 1.1. Let $X$ be a smooth variety and let $E$ be a coherent sheaf on $X$. Then $\operatorname{pd}_{\mathcal{O}_{x, X}}\left(E_{x}\right) \leq q$ if and only if for all $i>q$ we have

$$
\mathcal{E} x t_{\mathcal{O}_{X}}^{i}\left(E, \mathcal{O}_{X}\right)_{x}=0 .
$$

In particular, $E$ is locally free if and only if $\mathcal{E} x t_{\mathcal{O}_{X}}^{i}\left(E, \mathcal{O}_{X}\right)=0$ for all $i>0$.

Next, let us review definitions and results on reflexive sheaves on $\mathbf{P}_{\mathbb{K}}^{n}$. Reflexive sheaves form a category between torsion free sheaves and vector bundles.

Definition 1.1. We say a coherent sheaf $E$ on $\mathbf{P}_{\mathbb{K}}^{n}$ is reflexive if the canonical morphism $E \rightarrow E^{* *}$ is an isomorphism.

In this article, we use the following results on reflexive sheaves. For the proofs and details, see [3]. 
Proposition 1.2 ([3] , Proposition 1.3). A coherent sheaf $E$ on $\mathbf{P}_{\mathbb{K}}^{n}$ is reflexive if and only if $E$ is torsion free and $\operatorname{depth}_{\mathcal{O}_{x, \mathbf{P}_{\mathbb{K}}^{n}}}\left(E_{x}\right) \geq 2$ for all points $x \in \mathbf{P}_{\mathbb{K}}^{n}$ such that $\operatorname{dim} \mathcal{O}_{x, \mathbf{P}_{\mathbb{R}}^{n}} \geq 2$.

Corollary 1.3 ([3], Corollary 1.4$) \cdot \operatorname{codim}_{\mathbf{P}_{\mathbb{K}}^{n}} \operatorname{Sing}(E) \geq 3$ for a reflexive sheaf $E$ on $\mathbf{P}_{\mathbb{K}}^{n}$.

Proposition 1.4 ([3], Proposition 1.6). For a coherent sheaf $E$ on $\mathbf{P}_{\mathbb{K}}^{n}$, the following are equivalent:

1. $E$ is reflexive.

2. $E$ is torsion free and normal.

3. $E$ is torsion free and for each open set $U \subset \mathbf{P}_{\mathbb{K}}^{n}$ and each closed set $Z$ in $U$ satisfying $\operatorname{codim}_{U}(Z) \geq 2$, we have $\left.E\right|_{U} \simeq j_{*}\left(\left.E\right|_{U \backslash Z}\right)$, where $j: U \backslash Z \rightarrow U$ is an open immersion.

\section{The First proof of Theorem 0.2}

Let us prove Theorem 0.2. It suffices to show the "if" part of the statement. First, let us assume that $\operatorname{dim}(\operatorname{Sing}(E)) \geq 1$. Then any hyperplane $H \subset \mathbf{P}_{\mathbb{K}}^{n}$ inter$\operatorname{sects} \operatorname{Sing}(E)$. Take a point $x \in H \cap \operatorname{Sing}(E) \neq \emptyset$. Note that $\operatorname{depth}_{\mathcal{O}_{x, \mathbf{P}_{\mathbb{K}}^{n}}}\left(E_{x}\right) \leq$ $\operatorname{dim} \mathcal{O}_{x, \mathbf{P}_{\mathbb{K}}^{n}}-1$. Since the equation $h \in \mathcal{O}_{x, \mathbf{P}_{\mathbb{K}}^{n}}$ which defines $H$ at $x$ is a regu-

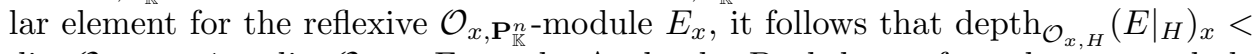
$\operatorname{dim} \mathcal{O}_{x, \mathbf{P}_{\mathbb{K}}^{n}}-1=\operatorname{dim} \mathcal{O}_{x, H}$. From the Auslander-Buchsbaum formula, we conclude that $\left.E\right|_{H}$ cannot even be locally free. Hence we may assume that $\operatorname{dim}(\operatorname{Sing}(E))=0$.

The next lemma is a generalization of Theorem 2.5 in $[3]$.

Lemma 2.1. Let $E$ be a reflexive sheaf on $\mathbf{P}_{\mathbb{K}}^{n}(n \geq 3)$ with $\operatorname{dim}(\operatorname{Sing}(E))=0$. Suppose the restriction $\left.E\right|_{H}$ to a hyperplane $H$ splits into a direct sum of line bundles. Then

$$
H^{1}\left(\mathbf{P}_{\mathbb{K}}^{n}, E(k)\right)=0, \text { for all } k \in \mathbb{Z}
$$

Proof of Lemma 2.1. We use the long exact sequence associated with the short exact sequence

$$
\left.0 \rightarrow E(k-1) \rightarrow E(k) \rightarrow E(k)\right|_{H} \rightarrow 0 .
$$

Because $\left.E(k)\right|_{H}$ is a direct sum of line bundles, it follows that $H^{1}\left(H,\left.E(k)\right|_{H}\right)=0$. So we have surjections

$$
H^{1}\left(\mathbf{P}_{\mathbb{K}}^{n}, E(k-1)\right) \rightarrow H^{1}\left(\mathbf{P}_{\mathbb{K}}^{n}, E(k)\right)(\forall k \in \mathbb{Z}) .
$$

To see that these cohomology groups are equal to zero, let us consider the spectral sequence of local and global Ext functors:

$$
E_{2}^{p, q}=H^{p}\left(\mathbf{P}_{\mathbb{K}}^{n}, \mathcal{E} x t_{\mathbf{P}_{\mathbb{K}}^{n}}^{q}(E, \omega)\right) \Rightarrow E^{p+q}=\operatorname{Ext}_{\mathbf{P}_{\mathbb{K}}^{n}}^{p+q}(E, \omega)
$$

where $\omega$ is the dualizing sheaf of $\mathbf{P}_{\mathbb{K}}^{n}$. The assumption $\operatorname{dim}(\operatorname{Sing}(E))=0$ implies $\operatorname{dim}\left(\operatorname{Supp}\left(\mathcal{E} x t_{\mathbf{P}_{\mathbb{K}}^{n}}^{q}(E, \omega)\right)\right)=0$ for all $q>0$. Thus it follows that $E_{2}^{p, q}=0$ unless $p=0$ or $q=0$. Moreover, Proposition 1.2 implies $\operatorname{depth}_{\mathcal{O}_{x, \mathbf{P}_{\mathbb{K}}^{n}}}\left(E_{x}\right) \geq 2$. From the Auslander-Buchsbaum formula, we have $\operatorname{pd}_{\mathcal{O}_{x, \mathbf{P}_{\mathbb{K}}^{n}}} E_{x}<n-1$ for all $x \in \mathbf{P}_{\mathbb{K}}^{n}$. It follows that $\mathcal{E} x t_{\mathbf{P}_{\mathbb{K}}^{n}}^{q}(E, \omega)=0$ for $\forall q \geq n-1$. Hence we have $E_{2}^{p, q}=0$ for $q \geq n-1$. Considering the convergence of this spectral sequence, we obtain the surjection

$$
\begin{array}{r}
H^{n-1}\left(\mathbf{P}_{\mathbb{K}}^{n}, \mathcal{H o m}_{\mathbf{P}_{\mathbb{K}}^{n}}(E, \omega)\right) \simeq H^{n-1}\left(\mathbf{P}_{\mathbb{K}}^{n}, E^{*} \otimes \omega\right) \\
\rightarrow \operatorname{Ext}_{\mathbf{P}_{\mathbb{K}}^{n}}^{n-1}(E, \omega) .
\end{array}
$$


Since $\operatorname{Ext}_{\mathbf{P}_{\mathbb{K}}^{n}}^{n-1}(E(k), \omega)$ is the Serre dual to $H^{1}\left(\mathbf{P}_{\mathbb{K}}^{n}, E(k)\right)$, they have the same dimension. From (2), we have

$$
\operatorname{dim} H^{1}\left(\mathbf{P}_{\mathbb{K}}^{n}, E(k)\right) \leq \operatorname{dim} H^{n-1}\left(\mathbf{P}_{\mathbb{K}}^{n}, E^{*}(-k) \otimes \omega\right)
$$

for all $k \in \mathbb{Z}$. The right hand side of (3) vanishes for $k \ll 0$. Then together with the surjectivity (1), we conclude that $H^{1}\left(\mathbf{P}_{\mathbb{K}}^{n}, E(k)\right)=0$, for all $k \in \mathbb{Z}$.

Now, let us put

$$
\left.E\right|_{H} \simeq \bigoplus_{i=1}^{r} \mathcal{O}_{H}\left(a_{i}\right)
$$

and $F:=\bigoplus_{i=1}^{r} \mathcal{O}_{\mathbf{P}_{\mathbb{K}}^{n}}\left(a_{i}\right)$. Noting that $\operatorname{Ext}_{\mathbf{P}_{\mathbb{K}}^{n}}^{1}(F, E(-1)) \simeq H^{1}\left(\mathbf{P}_{\mathbb{K}}^{n}, E\left(-a_{i}-1\right)\right)=$ 0 , Theorem 0.2 follows from the following theorem, which asserts that, roughly speaking, the structure of a reflexive sheaf can be recovered from its restriction to a hyperplane.

Theorem 2.2. Let $E$ and $F$ be reflexive sheaves on $\mathbf{P}_{\mathbb{K}}^{n}(n \geq 2)$ and $H$ be a hyperplane in $\mathbf{P}_{\mathbb{K}}^{n}$. Suppose $\left.\left.E\right|_{H} \cong F\right|_{H}$ and $\operatorname{Ext}_{\mathbf{P}_{\mathbb{K}}^{n}}^{1}(F, E(-1))=0$. Then $E \cong F$.

Proof of Theorem 2.2. We want to extend the isomorphism $\varphi:\left.\left.F\right|_{H} \rightarrow E\right|_{H}$ to one over $\mathbf{P}_{\mathbb{K}}^{n}$. That is possible since there is an exact sequence

$$
\begin{aligned}
0 & \rightarrow \operatorname{Hom}_{\mathbf{P}_{\mathbb{K}}^{n}}(F, E(-1)) \rightarrow \operatorname{Hom}_{\mathbf{P}_{\mathbb{K}}^{n}}(F, E) \\
& \rightarrow \operatorname{Hom}_{\mathbf{P}_{\mathbb{K}}^{n}}\left(F,\left.E\right|_{H}\right) \rightarrow \operatorname{Ext}_{\mathbf{P}_{\mathbb{K}}^{n}}^{1}(F, E(-1))=0,
\end{aligned}
$$

and every morphism $\left.\left.F\right|_{H} \rightarrow E\right|_{H}$ has a canonical extension to a morphism $F \rightarrow$ $\left.E\right|_{H}$. Let us fix an extended morphism $f: F \rightarrow E$ which satisfies $\left.f\right|_{H}=\varphi$. Now, let us consider the morphism $\operatorname{det} f: \operatorname{det} F \rightarrow \operatorname{det} E$. Since $\left.\left.E\right|_{H} \simeq F\right|_{H}$, ranks and first Chern classes of $E$ and $F$ are the same. Henceforth we can see that $\operatorname{det} f$ is a multiplication of some constant element in $\mathbb{K}$. Note that this constant is not zero. For $\operatorname{det} f$ is not zero on $H$. Thus at each point $x \in \mathbf{P}_{\mathbb{K}}^{n} \backslash(\operatorname{Sing}(E) \cup \operatorname{Sing}(F))$, the morphism $f_{x}$ is an isomorphism because at these points $f_{x}$ are the endomorphism of a direct sum of local rings of the same rank. Since $\operatorname{codim}_{\mathbf{P}_{\mathbb{K}}^{n}}(\operatorname{Sing}(E) \cup \operatorname{Sing}(F))>2$ and both of $E$ and $F$ are reflexive, the third condition of Proposition 1.4 implies that $f$ is also an isomorphism on $\mathbf{P}_{\mathbb{K}}^{n}$.

Remark 2.1. In Theorem 0.2, we cannot omit the assumption that $E$ is reflexive, i.e., "Restriction criterion" fails for torsion free sheaves. For example, consider the ideal sheaf $I_{p}$ on $\mathbf{P}_{\mathbb{K}}^{3}$ which corresponds to a closed point $p \in \mathbf{P}_{\mathbb{K}}^{3}$. Note that $I_{p}$ is not reflexive. Indeed, let us put $U=\mathbf{P}_{\mathbb{K}}^{3} \backslash\{p\}$ and $j: U \rightarrow \mathbf{P}_{\mathbb{K}}^{3}$ be an open immersion. It is easy to see that $\left.I_{p}\right|_{U} \simeq \mathcal{O}_{U}$. If $I_{p}$ is reflexive, then according to Proposition 1.4 $j_{*}\left(\left.I_{p}\right|_{U}\right) \simeq I_{p}$ must hold. However, clearly this is not true. Hence $I_{p}$ is not reflexive. Now, if we cut $I_{p}$ by a plane $H$ which does not contain $p$, then it is easily seen that $\left.I_{p}\right|_{H} \simeq \mathcal{O}_{H}$. However, of course, $I_{p}$ is not a line bundle on $\mathbf{P}^{3}$.

\section{THE SECOND PROOF}

Instead of Theorem 2.2, we can use the following result, which is the generalization of the famous Horrocks' splitting criterion (for example, see [5]). Combining this criterion with usual cohomological arguments and Lemma 2.1, we can give the second proof of Theorem 0.2 . 
Theorem 3.1. Let $\mathbb{K}$ be an algebraically closed field, $n$ be an integer greater than or equal to 2 , and $E$ be a coherent sheaf on $\mathbf{P}_{\mathbb{K}}^{n}$. Then $E$ splits into a direct sum of line bundles if and only if $H^{i}\left(\mathbf{P}_{\mathbb{K}}^{n}, E(k)\right)=0$ for all $k \in \mathbb{Z}, i=1, \cdots, n-1$, and $H^{0}\left(\mathbf{P}_{\mathbb{K}}^{n}, E(k)\right)=0$ for all $k \ll 0$.

Remark 3.1. Note that when $E$ is torsion free, then $H^{0}\left(\mathbf{P}_{\mathbb{K}}^{n}, E(k)\right)=0$ for all $k \ll 0$. This follows from the fact that all torsion free sheaves can be embedded into a direct sum of line bundles on $\mathbf{P}_{\mathbb{K}}^{n}$. So in the theorem, the condition $H^{0}\left(\mathbf{P}_{\mathbb{K}}^{n}, E(k)\right)=0$ is automatically satisfied for torsion free sheaves.

When $E$ is a vector bundle, Theorem 3.1] is just the splitting criterion of Horrocks. Thus for the proof of this theorem, it suffices to show the following lemma, which we can find in 1 .

Lemma 3.2. Let $X$ be a non-singular projective variety over an algebraically closed field $\mathbb{K}$ of dimension $n>1, L$ be an ample line bundle on $X$, and let $E$ be a coherent sheaf on $X$. Then $E$ is locally free if and only if $H^{i}(X, E(k))=0$ for all $k \ll 0$ and $i=0,1, \cdots, n-1$.

\section{ACKNOWLEDGEMEnTS}

The authors learned results of 93 from Professor F.-O. Schreyer. They are grateful to him. The authors also thank Takeshi Abe and Florin Ambro for many helpful comments and pointing out mistakes in the draft. The second author was supported by the JSPS Research Fellowship for Young Scientists.

\section{REFERENCES}

[1] R. Hartshorne, Ample subvarieties of algebraic varieties. Lecture Notes in Mathematics, 156, Springer-Verlag, Berlin, 1970. MR0282977(44:211)

[2] R. Hartshorne, Algebraic Geometry. Graduate Texts in Mathematics, 52, Springer-Verlag, 1977. MR0463157(57:3116)

[3] R. Hartshorne, Stable reflexive sheaves. Math. Ann. 254 (1980), 121-176. MR597077 (82b:14011)

[4] H. Matsumura, Commutative Algebra. W. A. Benjamin Co., New York, 1970. MR0266911 $(42: 1813)$

[5] C. Okonek, M. Schneider, H. Spindler, Vector Bundles on Complex Projective Spaces, Birkhäuser, Boston, MA, 1980. MR561910 (81b:14001)

[6] P. Orlik and H. Terao, Arrangements of hyperplanes. Grundlehren der Mathematischen Wissenschaften, 300, Springer-Verlag, Berlin, 1992. MR.1217488 (94e:52014)

[7] H. Terao, Generalized exponents of a free arrangement of hyperplanes and Shepherd-ToddBrieskorn formula. Invent. Math. 63 (1981), no. 1, 159-179. MR608532 (82e:32018b)

[8] M. Yoshinaga, Characterization of a free arrangement and conjecture of Edelman and Reiner. Invent. Math. 157 (2004), no. 2, 449-454. MR2077250 (2005d:52044)

Department of Mathematics, Hokkaido University, Sapporo 060-0810, Japan

E-mail address: abetaku@math.sci.hokudai.ac.jp

The Abdus Salam International Centre for Theoretical Physics, Strada Costiera

11, Trieste 34014, Italy

E-mail address: myoshina@ictp.it

Current address: Department of Mathematics, Kobe University, Kobe 657-8501, Japan

E-mail address: myoshina@math.kobe-u.ac.jp 